Article

\title{
The Classification of Inertinite Macerals in Coal Based on the Multifractal Spectrum Method
}

\author{
Man Liu ${ }^{1}$, Peizhen Wang ${ }^{1,2, *}$, Simin Chen ${ }^{1}$ and Dailin Zhang ${ }^{3}(\mathbb{D}$ \\ 1 School of Electrical and Information Engineering, Anhui University of Technology, \\ Ma'anshan 243002, China; lmdyne@163.com (M.L.); csmydd@163.com (S.C.) \\ 2 Key Laboratory of Metallurgical Emission Reduction \& Resources Recycling, Ministry of Education, \\ Anhui University of Technology, Ma'anshan 243002, China \\ 3 Anhui Key Laboratory of Clean Conversion and Utilization, Anhui University of Technology, \\ Ma'anshan 243002, China; agdzdl@ahut.edu.cn \\ * Correspondence: pzhwang@ahut.edu.cn
}

Received: 12 November 2019 ; Accepted: 8 December 2019; Published: 14 December 2019

check for updates

\begin{abstract}
Considering the heterogeneous nature and non-stationary property of inertinite components, we propose a texture description method with a set of multifractal descriptors to identify different macerals with few but effective features. This method is based on the multifractal spectrum calculated from the method of multifractal detrended fluctuation analysis (MF-DFA). Additionally, microscopic images of inertinite macerals were analyzed, which were verified to possess the property of multifractal. Simultaneously, we made an attempt to assess the influences of noise and blur on multifractal descriptors; the multifractal analysis was proven to be robust and immune to image quality. Finally, a classification model with a support vector machine (SVM) was built to distinguish different inertinite macerals from microscopic images of coal. The performance evaluation proves that the proposed descriptors based on multifractal spectrum can be successfully applied in the classification of inertinite macerals. The average classification precision can reach $95.33 \%$, higher than that of description method with gray level co-occurrence matrix (GLCM; about 7.99\%).
\end{abstract}

Keywords: coal; inertinite macerals; classification; multifractal analysis; support vector machine

\section{Introduction}

Macerals of coking coal closely relate to its characteristics, such as coke ability, caking ability, and thermal crushing performance, which directly influence the optical texture component distribution and quality of the coke [1-3]. Automatic classification and identification of different macerals in coal are of great significance for the effective evaluation of coal process properties [4]. Inertinite is one of the main groups in coal, and the classification of its macerals is of theoretical significance and application value for the efficient cleaning utilization of coal.

In view of the computational complexity, and the heavy workload, along with the subjective factors of the conventional manual and semi-manual method for maceral analysis, the methods of image processing and pattern recognition have been employed to analyze the components in coal $[5,6]$. Besides, based on the advantages of data analysis and processing, the machine learning approach is widely used in various fields [7]. Edward Lester [8] developed an image analysis technique to separate the major maceral groups of liptinite, vitrinite, fusinite, and semi-fusinite from the background resin according to the gray scales of the surface images captured with suitable camera exposure times. Nonetheless, even though the foregoing technique can work in some situations, it has not been implemented for a deep identification of macerals. There exists a fact that the characteristics of shape, color, contour, and texture of the microscopic image are essential for information expression of macerals 
in coal. Some related references have been published. To name a few, the authors of [9] completed the detection of approximately circular particles in the microscopic image of coal by the contour features, and the authors of [10] proposed a method to extract the outline of the maceral area by using structural elements. The texture features of local binary patterns (LBP) and the gray level co-occurrence matrix (GLCM) were combined to identify three major groups in coal macerals [11]. Grey scale, GLCM, Tamura, contourlet transform, and supervised locality preserving projections methods were employed by the authors in the previous work [12-15] to describe features of macerals. However, because the complex construction of macerals and similar morphological features among some different macerals exist, these techniques may not characterize them perfectly, especially for the features of texture.

In recent years, the fractal theory, first coined in [16], has been rapidly developed as a powerful analytical tool, which can reflect the heterogeneity and irregularities of a physical surface. There are several published techniques for characterizing the surface irregularity of coal with the mono-fractal method [17-21]. Nevertheless, it can not provide a comprehensive and accurate description of the details of image changes at different scales owing to the single scale of fractal dimensions. Coal's surface is known to be non-stationary and heterogeneous as a consequence of the long-term and multi-stage effects of geological processes. Some local trends in texture and dramatic changes in gray value are universal in microscopic images of macerals. Fortunately, a method named multifractal detrended fluctuation analysis (MF-DFA) can quickly eliminate local trends [22], making itself more suitable for describing the texture characteristics of the microscopic images of macerals. Given the superiority in solving non-stationary problems, the MF-DFA method has applications in quite a few fields [23-26]. Nevertheless, it was the first attempt that the approach was applied for the purpose of the classification of macerals in coal.

The major goal of our work was to find an artificial intelligence method to distinguish eight groups of inertinite macerals with few but stable and effective texture features. We analyzed and verified the multifractal properties of inertinite macerals by the method of MF-DFA. Additionally, multifractal descriptors of microscopic images were proposed based on the multifractal spectrum. In order to demonstrate the effectiveness of the multifractal descriptors, a comparison experiment of stability was implemented. Finally, we built an automatic classification model with support vector machine (SVM) to identify the inertinite macerals.

\section{Materials}

According to International Commission for Coal Petrology (ICCP) standard, coal is classified across three main maceral groups; i.e. vitrinite, liptinite, and inertinite [27]. Macerals of inertinite mainly come from woody fiber of plant or fungus [28]. The plant cellular structure of fusinite is relatively complete, and some of them have clear intercellular space and cellular wall. The cells of the pyrofusinite are crushed and shattered to present the shape of "arc" or "star-like", while the oxyfusinite has an unbroken cellular structure that exhibits a sieve shape. Semifusinite, the transitional maceral between telinite and fusinite, is located in the form of irregular strips. Secretinite is generally a product of silk carbonization reaction of secretions (tannin, resin, etc.), and few of them are derived from gelation of humus coal. Besides, the microscopic images are irregularly elliptical. Funginite is mainly derived from the remains of fungi or the secretions of higher plants, and has a honeycomb-like or reticulated multicellular structure inside. Additionally, the outer shape is flattened circular or ring-shaped due to extrusion. The cellular structure of the macrinite has a high protrusion and is generally an irregular matrix. A fragment of the inertinite group of particles have a particle size of less than $30 \mu \mathrm{m}$, angular or irregular in shape, and has no generally cellular structure. Most of the micrinites are distributed in asphaltene or mineral asphaltene with minor particle size and often small, nearly circular particles. Note that for fusinite, the two sub-macerals named pyrofusinite and oxyfusinite will be analyzed together with other six types of macerals in our work, as the texture differences are significant and obvious. 
From Figure 1, we can observe that there are some morphological differences among different macerals of inertinite in coal. However, their textures are fairly clear with singularity and conspicuous self-similarity. For such non-stationary structures, MF-DFA analysis can characterize them more effectively and show better processing power. In view of this, this paper performed the method of MF-DFA on each maceral image. For implementation, we used inertinite image data with 60 grayscale microscopic images of $227 \times 227$ pixels in size per group. The size was chosen to ensure that each image contained only one specific component, which is beneficial for subsequent feature extraction and classification experiments.

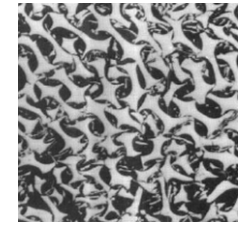

(a)

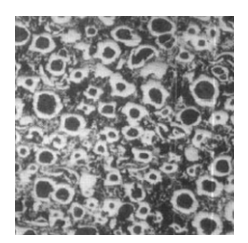

(e)

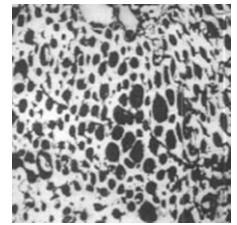

(b)

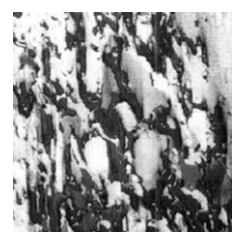

(f)

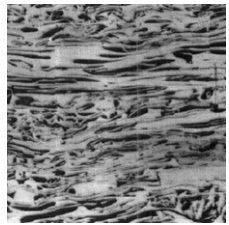

(c)

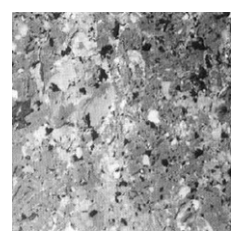

$(\mathrm{g})$

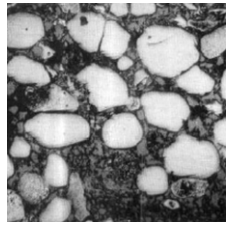

(d)

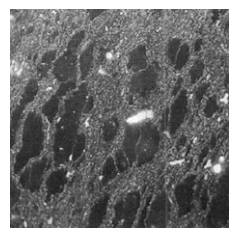

(h)

Figure 1. Typical microscopic images of inertinite in coal. (a) Pyrofusinite; (b) oxyfusinite; (c) semifusinit; (d) secretinite; (e) funginite; (f) macrinite; (g) inertodetrinite; (h) micirinite.

\section{Methods}

\subsection{Multifractal Spectrum Based on MF-DFA}

The method of MF-DFA is widely applied in scaling analysis due to its high accuracy and easy implementation. For grayscale images, it is not appropriate to calculate the multifractal spectrum with a gray series by the approach of one-dimensional MF-DFA. Generalizing the one-dimensional method to two-dimensional one can better express the information of the surface with self-similar properties [29]. Specifically, the process of calculating the multifractal spectrum of the grayscale image by using the two-dimensional MF-DFA method is determined as follows.

Step 1. Regard a microscopic image as a self-similar surface with a size of $M \times N$, which is represented by a matrix $X(i, j), i=1,2, \ldots, M$ and $j=1,2, \ldots, N$. Partition the surface into $M_{s} \times N_{s}$ $\left(M_{s} \equiv[M / s], N_{s} \equiv[N / s]\right)$ none-overlapping square subdomains of equal length $s$. Each subdomain is denoted by $X_{m, n}=X_{m, n}(i, j)$ with $X_{m, n}(i, j)=X(r+i, t+j)$ for $1 \leq i, j \leq s$ where $r=(m-1) s$, $t=(n-1) s$.

Step 2. For each subdomain $X_{m, n}$, the cumulative sum is constructed as follows

$$
G_{m, n}(i, j)=\sum_{k_{1}=1}^{i} \sum_{k_{2}=1}^{j} X_{m, n}\left(k_{1}, k_{2}\right)
$$

where $1 \leq i, j \leq s, m=1,2, \ldots, M_{s}, n=1,2, \ldots, N_{s}$. Note that $G_{m, n}=G_{m, n}(i, j)(i, j=1,2, . ., s)$ is a surface.

Step 3. The local trend $\tilde{G}_{m, n}$ for each surface $G_{m, n}$ can be obtained by fitting it with a pre-chosen bivariate polynomial function. In this paper, we adopt the trending function as 


$$
\tilde{G}_{m, n}(i, j)=a i+b j+c
$$

where $a, b$, and $c$ are free parameters to be estimated by the least-squares method. We can determine the residual matrix $y_{m, n}(i, j)$ with

$$
y_{m, n}(i, j)=G_{m, n}(i, j)-\tilde{G}_{m, n}(i, j) .
$$

Step 4. The detrended fluctuation $F(m, n, s)$ for each subdomain $X_{m, n}$ can be defined via the variance of $y_{m, n}(i, j)$ as follows

$$
F^{2}(m, n, s)=\frac{1}{s^{2}} \sum_{i=1}^{s} \sum_{j=1}^{s} y_{m, n}^{2}(i, j)
$$

Step 5 . The $q-t h$ order fluctuation is obtained by averaging over all the subdomain

$$
\begin{gathered}
F_{q}(s)=\exp \left\{\frac{1}{M_{s} N_{s}} \sum_{m=1}^{M_{s}} \sum_{n=1}^{N_{s}} \ln [F(m, n, s)]\right\}, q=0 \\
F_{q}(s)=\left[\frac{1}{M_{s} N_{s}} \sum_{m=1}^{M_{s}} \sum_{n=1}^{N_{s}}[F(m, n, s)]^{q}\right]^{1 / q}, q \neq 0 .
\end{gathered}
$$

Step 6. The scaling relation of the fluctuation can be determined by analyzing the $\log -\log F_{q}(s)$ versus the $s$ for different values of $s$ ranging from 6 to $(M, N) / 4$, which reads

$$
F_{q}(s) \propto s^{h(q)} .
$$

The scaling exponent $h(q)$ can be obtained by the linear regression of $\ln F_{q}(s)$ to $\ln s$, which is also called the generalized Hurst index. For each $q$, the corresponding traditional scaling exponent as $\tau(q)$

$$
\tau(q)=q h(q)-D_{f}
$$

Note that, $D_{f}$ represents the fractal dimension of the geometric support. For the two-dimensional microscopic image of this paper, we take the value of $D_{f}=2$.

Step 7. The multifractal surface can be characterized by Hölder exponent $\alpha(q)$ and singularity spectrum $f(\alpha)$, which are given by the Legendre transform [30].

$$
\begin{gathered}
\alpha(q)=\tau^{\prime}(q)=h(q)+q h^{\prime}(q) \\
f(\alpha)=q \alpha(q)-\tau(q)=q[\alpha-h(q)]+2 .
\end{gathered}
$$

Here, the multifractal singularity spectrum $f(\alpha)$ is a continuous exponential spectrum used to characterize multiple fractal sets, which provides a complete statistical description of the internal inconsistencies of fractals.

\subsection{Multifractal Analysis and Feature Extraction}

We express grayscale images of inertinite macerals as two-dimensional matrices and analyze them in accordance with the multifractal detrended fluctuation analysis introduced previously. It is worth mentioning that in the partitioning process, the upper-right and bottom areas are ignored since the image sizes of $M$ and $N$ are not particular multiples of the small square $s$. Hence, we can repeat the partitioning process in the other three directions. Taking the typical microscopic images in Figure 1 
as examples, we calculate the scaling exponent $h(q)$ with different values of $q$ in the range from -6 to 6; then, the corresponding function $\tau(q)$ can be obtained according to Equation (8). The result $\tau(q)$ is given in Figure 2, and the inset displays the scaling exponent $h(q)$. We can find that the function $\tau(q)$ is nonlinear with respect to $q$, which indicates that the exponent $\tau(q)$ is dependent on $q$. Nonlinearity also confirms that the microscopic images of inertinite do possess multifractal nature.

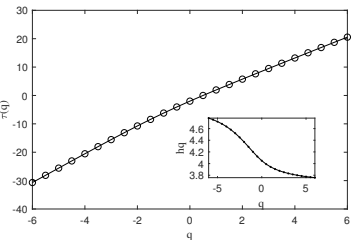

(a)

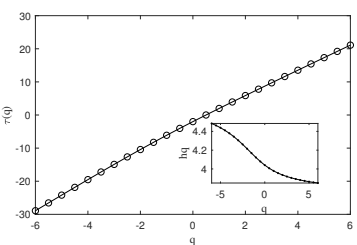

(e)

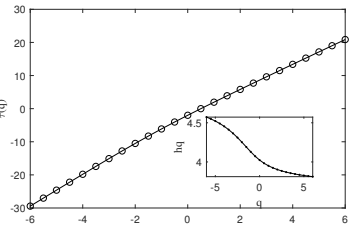

(b)

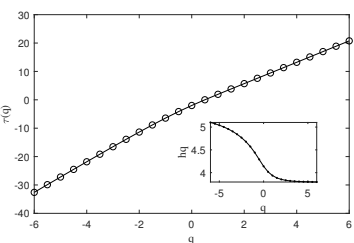

(f)

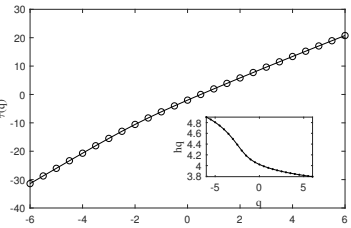

(c)

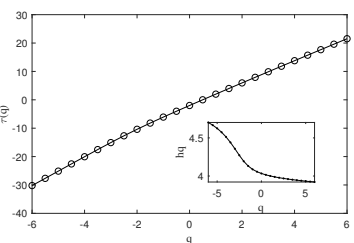

$(\mathrm{g})$

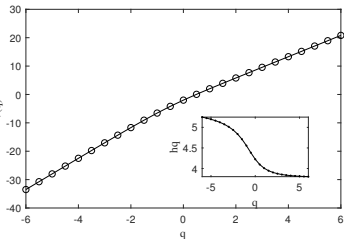

(d)

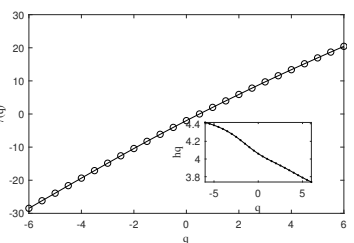

(h)

Figure 2. Dependence of $\tau(q)$ and $h(q)$ on $q$ for the typical microscopic images of inertinite macerals. (a) Pyrofusinite; (b) oxyfusinite; (c) semifusinit; (d) secretinite; (e) funginite; (f) macrinite; (g) inertodetrinite; (h) micirinite.

According to Equations (9) and (10), we calculate the multifractal spectra of the macerals of inertinite, which are displayed in Figure 3. Their graphs are typically barbed, indicating that different parts with different singularities have different fractal dimensions, confirming the multifractal properties of our microscopic images. The multifractal singularity spectrum is a single-peak map normally, and several important multifractal feature parameters can be extracted as the texture descriptors of the corresponding image, such as the minimum value of the local singularity $\alpha_{\min }$, the maximum value of the local singularity $\alpha_{\max }$, and the maximum value of the spectrum $f_{\max }$.

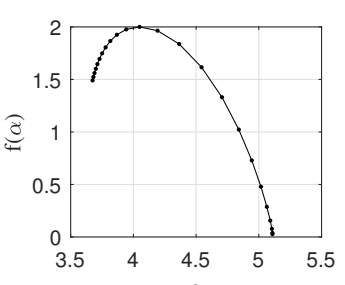

(a)

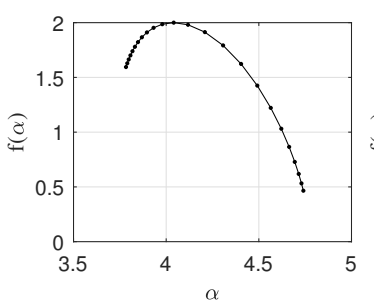

(e)

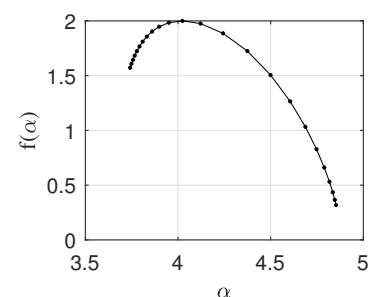

(b)

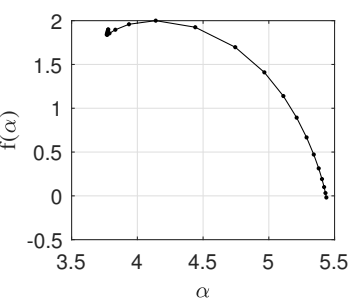

(f)

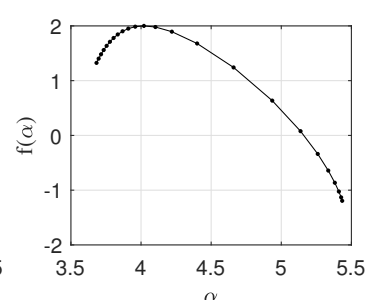

(c)

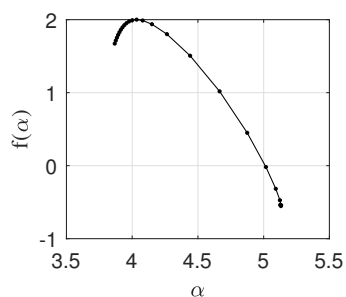

(g)

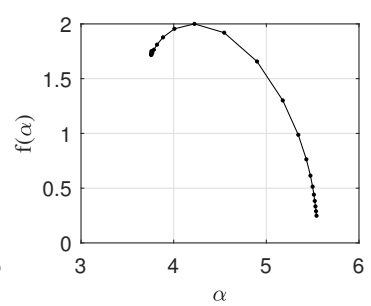

(d)

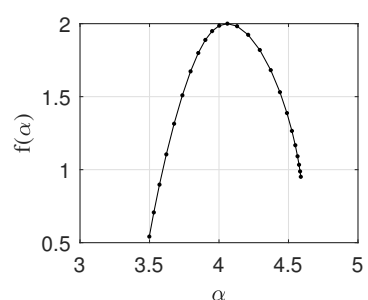

(h)

Figure 3. Multifractal spectra of microscopic images of the typical inertinite macerals. (a) Pyrofusinite; (b) oxyfusinite; (c) semifusinite; (d) secretinite; (e) funginite; (f) macrinite; (g) inertodetrinite; (h) micirinite. 
Additionally, the multifractal descriptors of $\alpha_{\min }, \alpha_{\max }$, and $f_{\max }$ are used to build a threedimensional space to test the distinguishing ability of each of the two groups. We calculated the multifractal spectra of 480 grayscale images in the inertinite data set, and their corresponding multifractal descriptors are plotted in Figure 4, respectively. We can find that it is not difficult to distinguish different groups due to the fact that the same components are clustered together and different macerals are separated in the space. It is worth mentioning that a small number of combinations of macerals have a certain degree of overlap due to a high similarity between their textures. However, the majority of combinations are separable in our three-dimensional space.

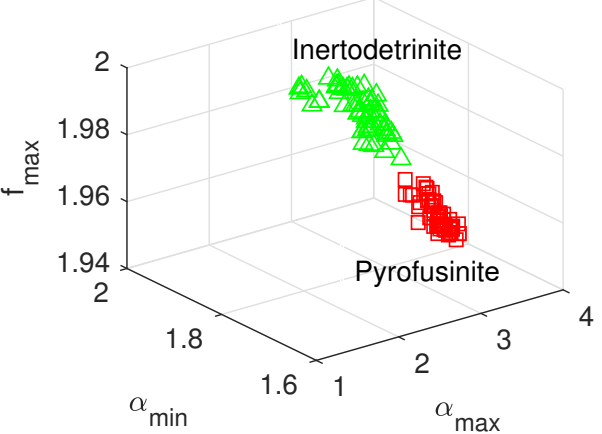

(a)

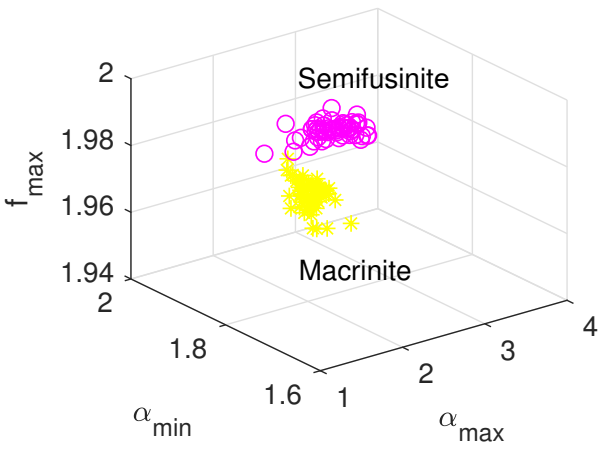

(c)

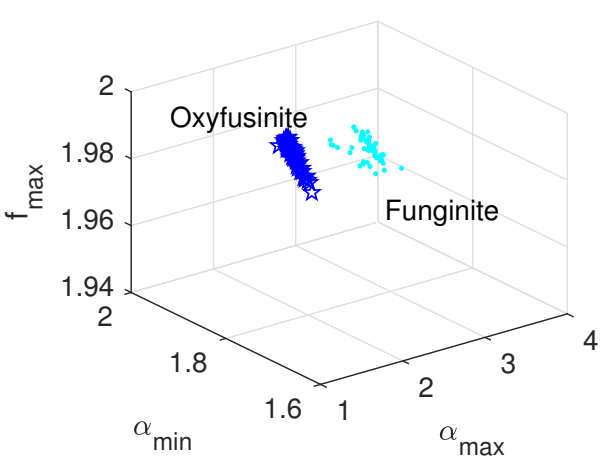

(b)

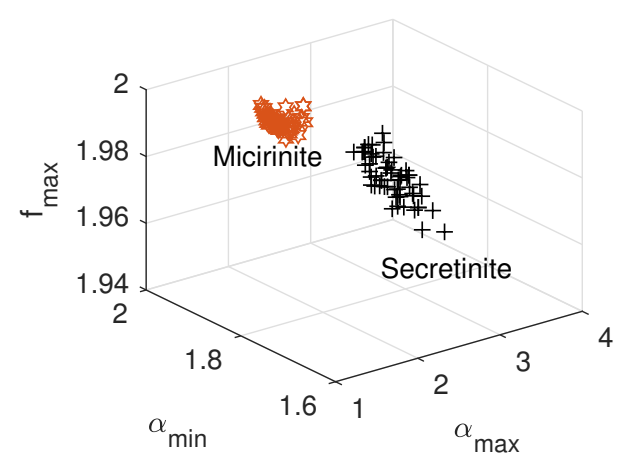

(d)

Figure 4. The three-dimensional space with multifractal descriptors for every pair of groups of intertinite macerals. (a) inertodetrinite Vs pyrofusinite; (b) oxyfusinite Vs funginite; (c) semifusinite Vs macrinite; (d) micrinite Vs secretinite.

\section{Stability Analysis of Multifractal Feature Descriptors}

The feature descriptors $\left(\alpha_{\min }, \alpha_{\max }, f_{\max }\right)$ extracted from the multifractal spectrum should be able to characterize the significant textural information of the inertinite macerals. As is well known, effective texture features for image recognition are supposed to be robust and not subject to image quality. In this section, we consider the stability of our multifractal descriptors in terms of noise immunity and anti-blurring ability, and then illustrate the superiority of multifractal descriptors compared to the traditional feature descriptors.

\subsection{Stability to Image Noise}

Textural features of images can be disturbed by noise to a great extent. In this paper, Gaussian noise, speckle noise, and salt and pepper noise are added to the inertinite microscopic images to investigate the influence of noise on the multifractal spectrum. Figure 5 gives the images of pyrofusinite 
with the addition of Gaussian noise (0-mean and variance of 0.05), speckle noise (variance of 0.05), and salt and pepper noise (density of $2 \%$ ), respectively.

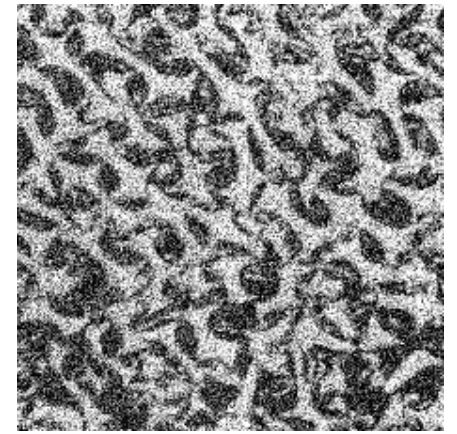

(a)

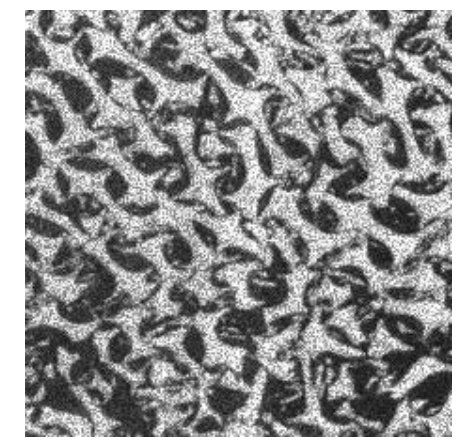

(b)

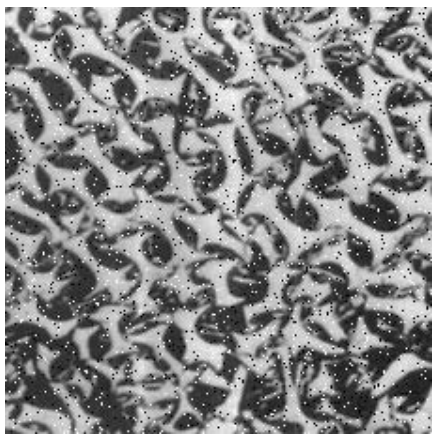

(c)

Figure 5. Typical images of pyrofusinite with different noises. (a) Gaussian noise; (b) speckle noise; (c) salt and pepper noise.

The multifractal spectra $f(\alpha)$ of the pyrofusinite images with various noises were calculated and the comparisons with the original image were done. As shown in Figure 6, the spectrum of pyrofusinite image with speckle noise is almost identical to the original one, which illustrates that the speckle noise has a slight influence on pyrofusinite image. In addition, we extract and report the values of multifractal descriptors of eight groups of inertinite macerals with different noises in Table 1. For comparisons, the statistical features, such as angular second moment (ASM), entropy, moment of inertia (IM), and correlation based on GLCM, were calculated and listed in Table 2. From Tables 1 and 2, we can find that the multifractal descriptors are relatively stable, while the GLCM-based texture descriptors are sensitive to noise, and the value of IM fluctuates significantly with different noises.

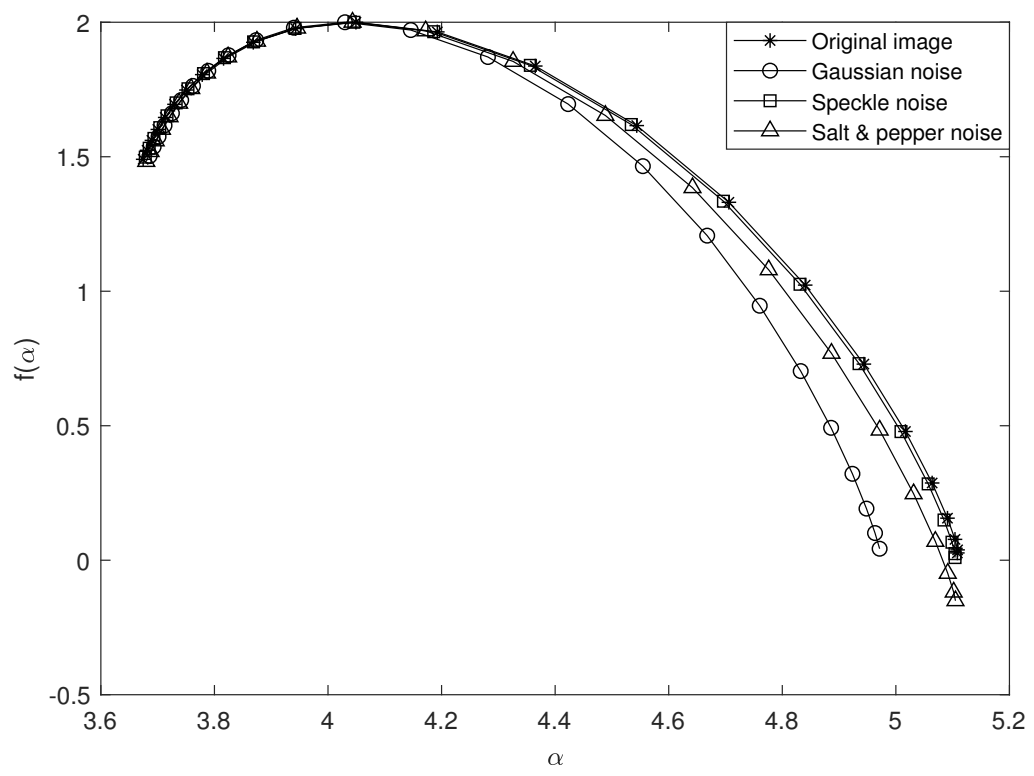

Figure 6. Multifractal spectra of the microscopic images of pyrofusinite with different noises. 
Table 1. Multifractal descriptors of typical microscopic images of inertinite with different noises. Sample labels (a)-(h) are consistent with the labels of typical images in Figure 1.

\begin{tabular}{cccccccccccccc}
\hline \multirow{2}{*}{$\begin{array}{c}\text { Sample } \\
\text { Label }\end{array}$} & \multicolumn{3}{c}{ Original Image } & \multicolumn{3}{c}{ Gaussian Noise } & \multicolumn{3}{c}{ Speckle Noise } & \multicolumn{3}{c}{ Salt \& Pepper Noise } \\
\cline { 2 - 12 } & $\alpha_{\min }$ & $\alpha_{\max }$ & $f_{\max }$ & $\alpha_{\min }$ & $\boldsymbol{\alpha}_{\max }$ & $f_{\max }$ & $\alpha_{\min }$ & $\alpha_{\max }$ & $f_{\max }$ & $\alpha_{\min }$ & $\alpha_{\max }$ & $f_{\max }$ \\
\hline (a) & 1.8717 & 2.4682 & 1.9974 & 1.8776 & 2.3777 & 1.9978 & 1.8731 & 2.4640 & 1.9975 & 1.8760 & 2.4406 & 1.9976 \\
(b) & 1.9019 & 2.3411 & 1.9982 & 1.9066 & 2.3093 & 1.9985 & 1.9101 & 2.3239 & 1.9984 & 1.9060 & 2.3163 & 1.9984 \\
(c) & 1.8987 & 2.5650 & 1.9985 & 1.8986 & 2.2927 & 1.9987 & 1.8970 & 2.5879 & 1.9985 & 1.9018 & 2.3932 & 1.9987 \\
(d) & 1.8810 & 2.7293 & 1.9946 & 1.8879 & 2.4767 & 1.9959 & 1.8869 & 2.7477 & 1.9948 & 1.8825 & 2.5918 & 1.9953 \\
(e) & 1.9213 & 2.2807 & 1.9985 & 1.9221 & 2.2545 & 1.9987 & 1.9211 & 2.2808 & 1.9986 & 1.9240 & 2.2550 & 1.9987 \\
(f) & 1.8868 & 2.7113 & 1.9948 & 1.8959 & 2.4883 & 1.9961 & 1.8926 & 2.7285 & 1.9952 & 1.8881 & 2.6409 & 1.9954 \\
(g) & 1.9615 & 2.4384 & 1.9992 & 1.9607 & 2.2565 & 1.9993 & 1.9635 & 2.4191 & 1.9992 & 1.9608 & 2.3784 & 1.9993 \\
(h) & 1.8924 & 2.2427 & 1.9986 & 1.9075 & 2.2099 & 1.9989 & 1.9003 & 2.2446 & 1.9986 & 1.9023 & 2.2149 & 1.9988 \\
\hline
\end{tabular}

To further clarify that our multifractal descriptors of $\alpha_{\min }, \alpha_{\max }$ and $f_{\max }$ possess better anti-noise stability than that of GLCM-based texture parameters, the average relative errors of feature descriptors from typical microscopic images with different noises are calculated and displayed in Figure 7 . We can see that three types of noises all have a great influence on GLCM-based texture parameters, especially for the moment of inertia, whose relative error is much higher than $100 \%$ for each maceral of inertinite. The parameter of IM of secretinite is highly sensitive to different noise with a relative error close to $400 \%$. Furthermore, the relative errors of the three multifractal texture descriptors are particularly low among the seven parameters, none of which exceeds $15 \%$, and that of $f_{\max }$ even closes in on zero. From the results of noise immunity experiment, it is clear that our descriptors of $\alpha_{\min }, \alpha_{\max }$, and $f_{\max }$ possess great stability to various noises.
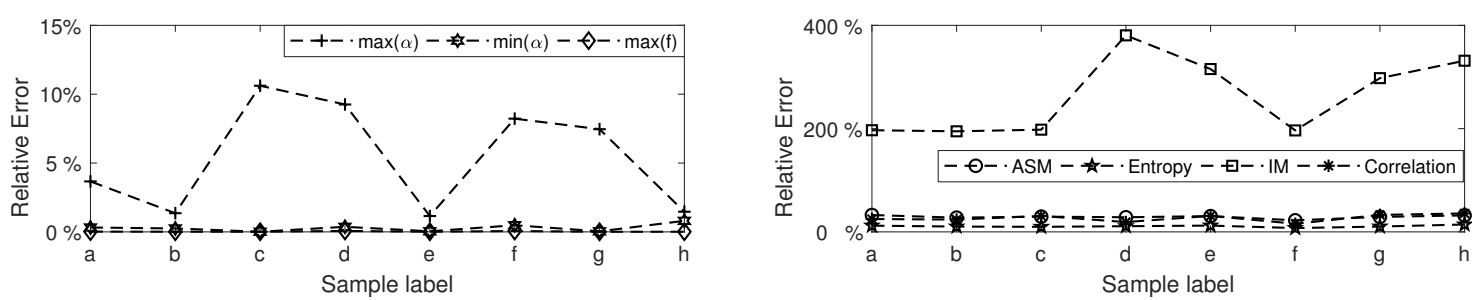

(a)
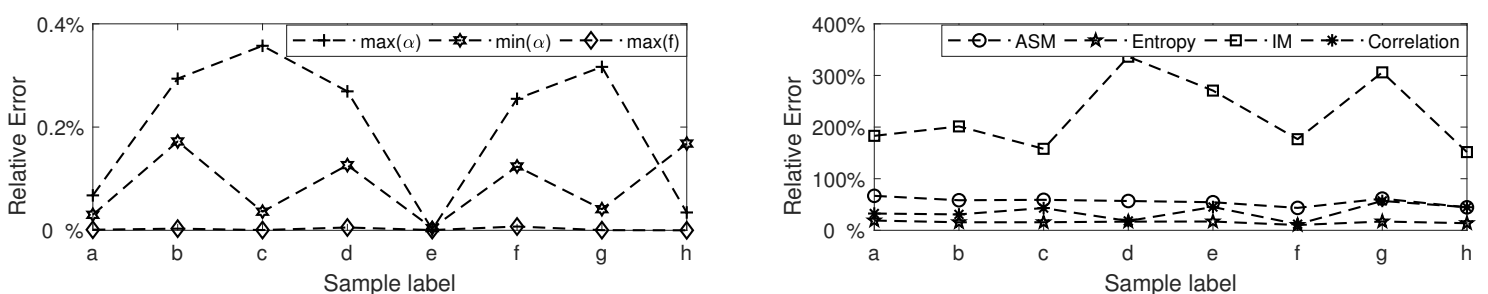

(b)
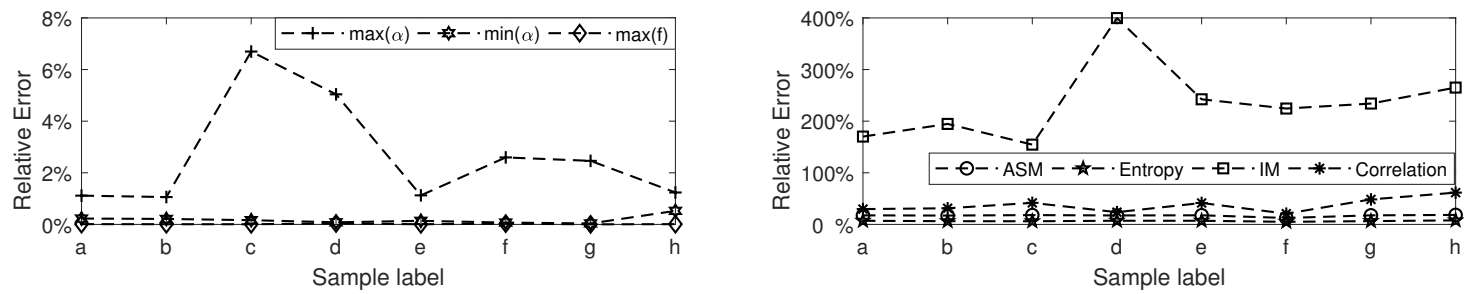

(c)

Figure 7. Relative errors of texture descriptors of typical inertinite microscopic images with different noises. (a) Gaussian noise; (b) speckle noise; (c) salt and pepper noise. 
Table 2. GLCM-based descriptors of typical microscopic images of inertinite with different noises. Sample labels (a)-(h) are consistent with the labels of typical images in Figure 1.

\begin{tabular}{|c|c|c|c|c|c|c|c|c|c|c|c|c|c|c|c|c|}
\hline \multirow{2}{*}{$\begin{array}{l}\text { Sample } \\
\text { Label }\end{array}$} & \multicolumn{4}{|c|}{ Original Image } & \multicolumn{4}{|c|}{ Gaussian Noise } & \multicolumn{4}{|c|}{ Speckle Noise } & \multicolumn{4}{|c|}{ Salt \& Pepper Noise } \\
\hline & ASM & Entropy & IM & Correlation & ASM & Entropy & $\mathrm{IM}$ & Correlation & ASM & Entropy & IM & Correlation & ASM & Entropy & IM & Correlation \\
\hline (a) & 0.0311 & 4.1129 & 3.8208 & 0.0583 & 0.0057 & 5.3468 & 22.6266 & 0.0221 & 0.0103 & 4.8912 & 10.8139 & 0.0393 & 0.0257 & 4.4021 & 10.3217 & 0.0410 \\
\hline (b) & 0.0275 & 4.1562 & 3.6523 & 0.0577 & 0.0086 & 5.2229 & 21.4380 & 0.0233 & 0.0115 & 4.8042 & 11.0043 & 0.0399 & 0.0228 & 4.4171 & 10.7620 & 0.0396 \\
\hline (c) & 0.0196 & 4.3374 & 4.0526 & 0.0813 & 0.0051 & 5.3947 & 24.1092 & 0.0187 & 0.0160 & 4.6026 & 10.3152 & 0.0474 & 0.0080 & 5.0158 & 10.4514 & 0.0462 \\
\hline (d) & 0.0280 & 4.0889 & 1.8521 & 0.0467 & 0.0082 & 5.2103 & 19.4783 & 0.0245 & 0.0121 & 4.7789 & 8.0808 & 0.0382 & 0.0231 & 4.3789 & 9.2544 & 0.0356 \\
\hline (e) & 0.0212 & 4.1389 & 2.6026 & 0.0895 & 0.0050 & 5.3962 & 23.1238 & 0.0203 & 0.0097 & 4.8445 & 9.6475 & 0.0490 & 0.0175 & 4.4307 & 8.9111 & 0.0523 \\
\hline (f) & 0.0239 & 4.3333 & 3.2983 & 0.0395 & 0.0105 & 5.1325 & 19.5031 & 0.0240 & 0.0135 & 4.7792 & 9.1217 & 0.0348 & 0.0209 & 4.5520 & 10.7023 & 0.0313 \\
\hline (g) & 0.0212 & 4.2299 & 2.7384 & 0.1043 & 0.0059 & 5.3177 & 23.1227 & 0.0186 & 0.0082 & 4.9513 & 11.1185 & 0.0449 & 0.0175 & 4.4992 & 9.1495 & 0.0538 \\
\hline (h) & 0.0327 & 3.8546 & 2.4882 & 0.1421 & 0.0066 & 5.2311 & 23.1091 & 0.0153 & 0.0181 & 4.3865 & 6.2596 & 0.0786 & 0.0267 & 4.1566 & 9.0840 & 0.5460 \\
\hline
\end{tabular}




\subsection{Stability to Image Blurring}

Due to the limitations of objective conditions and the interference from human factors, some inevitable phenomena may occur in the process of microscopic image acquisition, such as motion blur caused by lens jitter and defocusing blur caused by inaccurate focusing, while stable texture parameters should have good immunity to these kinds of fuzzy degradation [31].

Taking the image of pyrofusinite as an example, motion blur and defocusing blur degradation are processed as shown in Figure 8. We plot their multifractal spectra in Figure 9 and compare the spectra with the original one. As can be seen from the multifractal spectra, after image blurring, the value of $f_{\max }$ fluctuates slightly between 2 and 2.05, indicating the extracted texture parameter $f_{\max }$ is not sensitive to blurring.

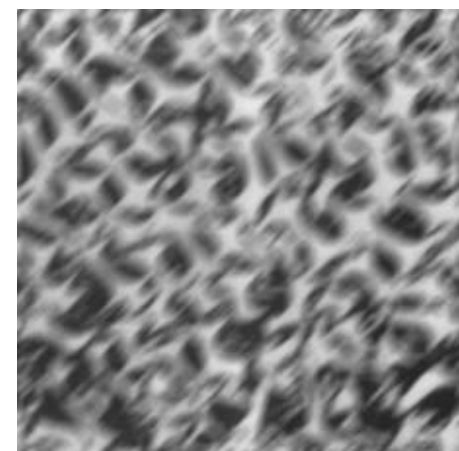

(a)

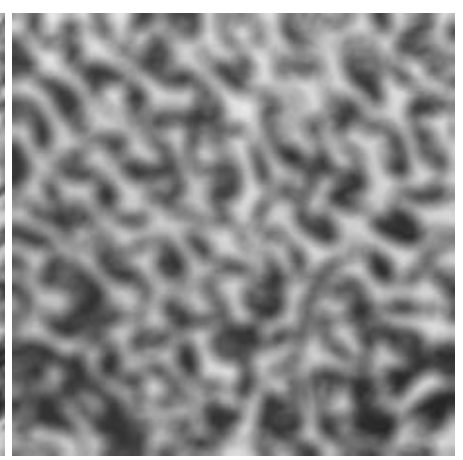

(b)

Figure 8. Typical images of pyrofusinite with different blurred processing methods. (a) Motion blurred image; (b) defocus blurred image.

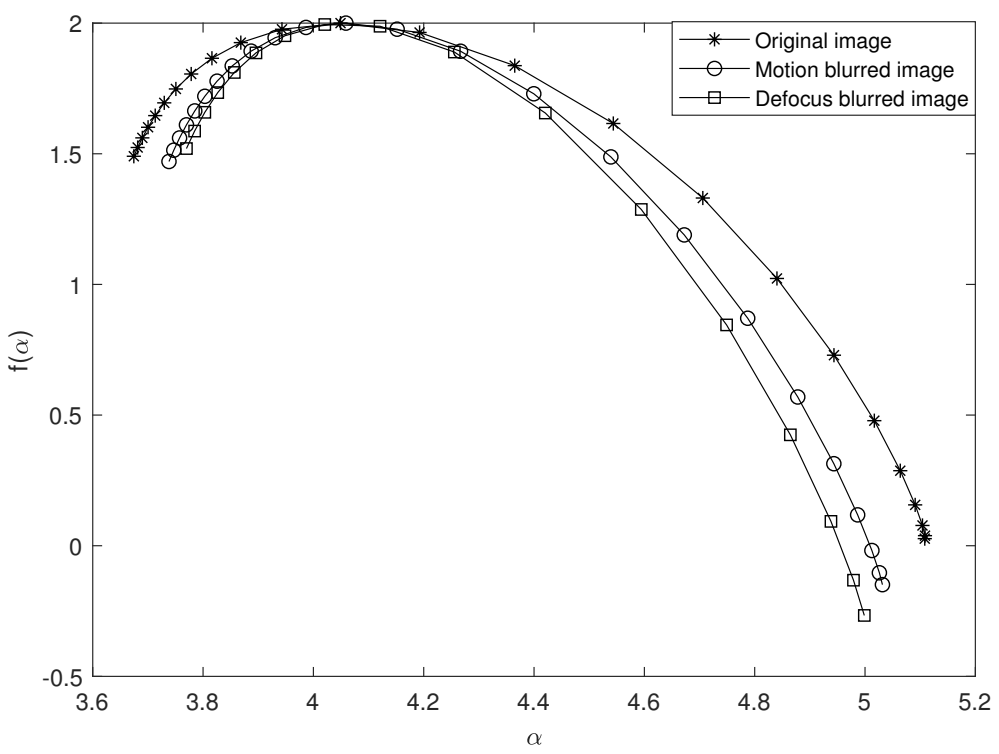

Figure 9. Multifractal spectra of the microscopic images of pyrofusinite with different blurring.

In order to demonstrate the robustness of the multifractal descriptors to image blurring more convincingly, we perform motion blur and defocus blur on all microscopic images labeled (a)-(h) form Figure 1 . Then the parameters of $\alpha_{\min }, \alpha_{\max }$, and $f_{\max }$ are calculated; besides, the GLCM-based texture features are also calculated for comparison. Figure 10 shows the average error of these texture features. For eight types of inertinite macerals, the relative error of $f_{\max }$ is close to $0, \alpha_{\min }$ and $\alpha_{\max }$ are between $0 \%$ and $15 \%$, which indicates that the multifractal features have excellent robustness and are insensitive to blurring. However, GlCM-based features are susceptible to image blurring. For example, 
the relative error of the second-order moment of the semifusinite with defocus blurring is even higher than $300 \%$; the parameter of energy is relatively stable in the microscopic images of inertinite macerals, all of which are less than $40 \%$. The above analysis depicts that the three multifractal descriptors possess great stability to different kinds of blurring.
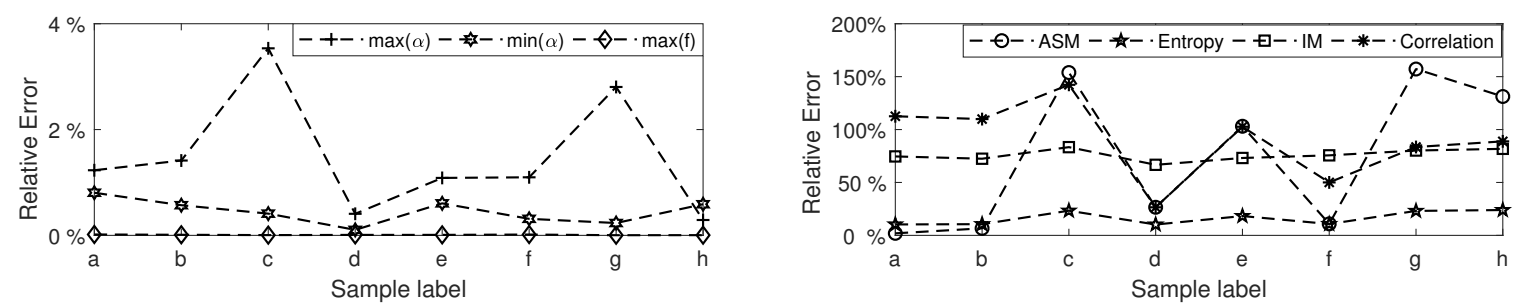

(a)
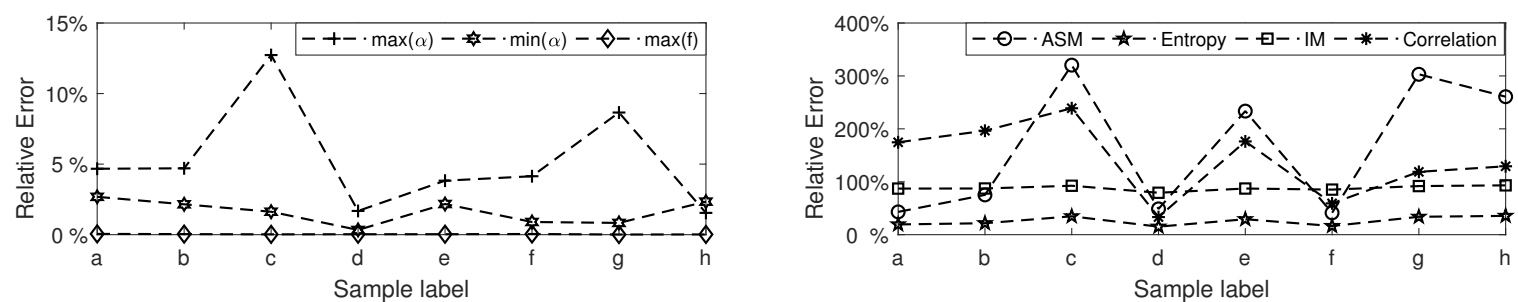

(b)

Figure 10. The relative errors of textural descriptors of typical inertinite microscopic images with different blurring types. (a) Motion blurred image; (b) defocus blurred image.

\section{Classification Experiment}

\subsection{Experiment Design}

Considering small samples, SVM is employed to build a classifier for the classification of inertinite macerals [32]. To address the non-linear and the multi-classes problem in this paper, the input data are mapped into high-dimensional space with a non-linear mapping, and the relevant classification function can be expressed as

$$
\mathrm{F}(x)=\operatorname{sgn}\left[\sum_{i=1}^{n} a_{i} y_{i} K\left(x_{i}, x\right)+\mathrm{b}_{0}\right],
$$

where $a_{i}, i=1, \ldots, n$ are Lagrange multipliers, the class to which a sample is assigned is labeled $y_{i}$, and $K\left(x_{i}, x\right)$ represents a kernel function, which is the radial basis function (RBF) kernel function here.

The classification model for inertinite macerals with the SVM-based classifiers is illustrated in Figure 11. To implement the multi-classification, we construct a classifier group with 28 RBF-SVM classifiers to distinguish eight groups of inertinite macerals based on the one-against-one (1A1) technique and optimize the error parameter (usually designated $c$ ) and parameter $\gamma$ in RBF kernel function by a grid search [33,34]. Besides, 40 of the microscopic samples per group are used for training, and the remaining 20 samples for testing and each classifier is used to distinguish two different classes of inertinite macerals. Then, the remaining testing samples per group are input into the trained classifiers. The specific testing process is as follows.

Step 1. Calculate the texture descriptors of $\alpha_{\min }, \alpha_{\max }$ and $f_{\max }$ for each image in the testing set.

Step 2. Input the texture data obtained in the previous step into the classifier group in turn.

Step 3. Count the votes in eight groups; the testing image is classified into the group with the best poll numbers. 
Step 4. Repeat the above steps for the remaining images, and finally, get the category for each training images.

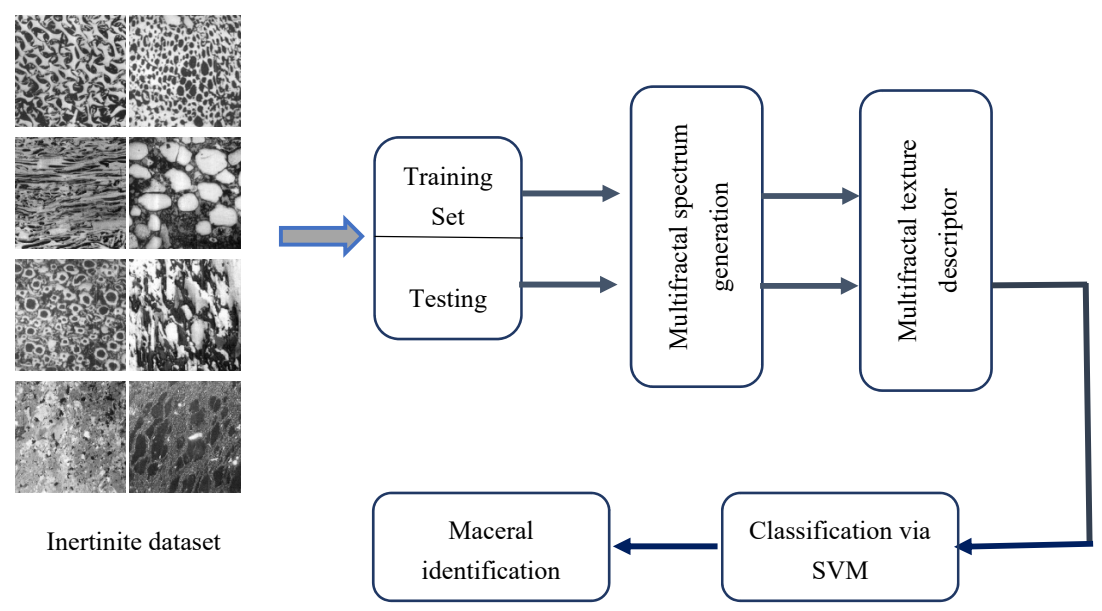

Figure 11. Classification model for inertinite macerals with the SVM-based classifiers.

\subsection{Evaluation Measures}

The results of the automatic classification method are quantitatively evaluated by ensemble of popular measures. The measures used in our work comprise precision, recall, and F-measure.

The class agreement of the predicted labels with the positive labels given by the classifier is estimated by precision, and the validity of the positive label recognition is measured by recall. The F-measure is defined as a scaled harmonic mean of precision and recall.

$$
\begin{gathered}
\text { precision }_{i}=\frac{t p_{i}}{t p_{i}+f p_{i}} \\
\text { recall }_{i}=\frac{t p_{i}}{t p_{i}+f n_{i}} \\
\text { F-measure }_{i}=\frac{2 * \text { precision }_{i} * \text { recall }_{i}}{\text { precision }_{i}+\text { recall }_{i}},
\end{gathered}
$$

where $t p_{i}, f p_{i}, t n_{i}$, and $f n_{i}$ denote the values of true positives, false positives, true negatives, and false negatives for class $i$, respectively. Using the above measurements, the performance of proposed classification model can be conducted for comparison purposes. Additionally, for the purpose of comprehensively evaluating the average performance of eight groups of inertinite macerals, we consider the average values of precision (macro-precision), the average values of recall (macro-recall), and macro-F, which is a scaled harmonic mean of macro-precision and macro-recall.

\subsection{Experimental Results}

Based on the classification model, each RBF-SVM classifier is trained with the training samples to get specific values of parameters $c$ and $\gamma$, as summarized in Table 3. For the testing samples, the previous evaluation performance of classifying inertinite macerals using multifractal descriptors is reported in Table 4. For each maceral, the classification result has achieved satisfactory performance in terms of precision, recall, and F-measure. We notice that the precision performances of oxyfusinite, secretinite, and funginite are slightly lower than those of the best performances of about 0.1304 , 0.9520 , and 0.1000 , which may be due to their fractal similarity corresponding to the distribution of multifractal spectra, as shown in Figure 3. Remarkably, the result for macrinite presents the most 
appealing performance with three full marks. This may be attributed to the fact that the MF-DFA method can effectively eliminate the local trends of non-stationary images and detect their multifractal features more accurately. These data from the evaluation matrices indicate that our multifractal features are effective in representing texture information of microscopic images of inertinite macerals.

Table 3. Objects and parameters of different classifiers. (a) Pyrofusinite; (b) oxyfusinite; (c) semifusinite; (d) secretinite; (e) funginite; (f) macrinitee; (g) inertodetrinite; (h) micirinite.

\begin{tabular}{cccccccc}
\hline Classifier & Objects & $c$ & $\gamma$ & Classifier & Objects & $c$ & $\gamma$ \\
\hline RBF-SVM1 & (a) Vs (b) & 0.5000 & 2.0000 & RBF-SVM15 & (c) Vs (e) & 0.0313 & 0.0313 \\
RBF-SVM2 & (a) Vs (c) & 0.0313 & 0.0313 & RBF-SVM16 & (c) Vs (f) & 0.0313 & 0.0313 \\
RBF-SVM3 & (a) Vs (d) & 0.2500 & 32.0000 & RBF-SVM17 & (c) Vs (g) & 0.0313 & 0.0313 \\
RBF-SVM4 & (a) Vs (e) & 4.0000 & 32.0000 & RBF-SVM18 & (c) Vs (h) & 0.0313 & 0.0313 \\
RBF-SVM5 & (a) Vs (f) & 16.0000 & 32.0000 & RBF-SVM19 & (d) Vs (e) & 2.0000 & 16.0000 \\
RBF-SVM6 & (a) Vs (g) & 0.0313 & 2.0000 & RBF-SVM20 & (d) Vs (f) & 0.0313 & 0.0313 \\
RBF-SVM7 & (a) Vs (h) & 1.0000 & 32.0000 & RBF-SVM21 & (d) Vs (g) & 0.0313 & 0.5000 \\
RBF-SVM8 & (b) Vs (c) & 0.0313 & 0.0313 & RBF-SVM22 & (d) Vs (h) & 0.0313 & 8.0000 \\
RBF-SVM9 & (b) Vs (d) & 0.0313 & 0.0313 & RBF-SVM23 & (e) Vs (f) & 0.0313 & 32.0000 \\
RBF-SVM10 & (b) Vs (e) & 0.0625 & 8.0000 & RBF-SVM24 & (e) Vs (g) & 0.0313 & 0.0313 \\
RBF-SVM11 & (b) Vs (f) & 0.0313 & 0.0313 & RBF-SVM25 & (e) Vs (h) & 16.0000 & 2.0000 \\
RBF-SVM12 & (b) Vs (g) & 0.0313 & 1.0000 & RBF-SVM26 & (f) Vs (g) & 0.0313 & 0.5000 \\
RBF-SVM13 & (b) Vs (h) & 0.0313 & 0.2500 & RBF-SVM27 & (f) Vs (h) & 0.0313 & 32.0000 \\
RBF-SVM14 & (c) Vs (d) & 0.0313 & 0.2500 & RBF-SVM28 & (g) Vs (h) & 0.0313 & 0.0313 \\
\hline
\end{tabular}

As a comparison, the performance evaluation of the classification of GLCM-based descriptors is reported in Table 5. It is not surprising to find that the GLCM-based descriptors always lead to unsatisfactory performance when compared to multifractal descriptors. This may be explained by the fact that the statistical features based on GLCM are not applicable for describing texture images with complex and heterogeneous naturals. Especially for the maceral of inertodetrinite, the three evaluation values are as low as $0.667,0.5000$, and 0.667 , nearly half of the corresponding evaluation values of our method, which are far from satisfying our classification purpose. Overall, we give the average performance evaluation in Figure 12. The macro-precision of the GLCM-based descriptors can be improved by means of the proposed multifractal descriptors up to $7.99 \%$. This holds in both micro-recall and macro-F with improvements of $10.00 \%$ and $9.02 \%$, respectively. These data present report the effectiveness and feasibleness of our proposed method.

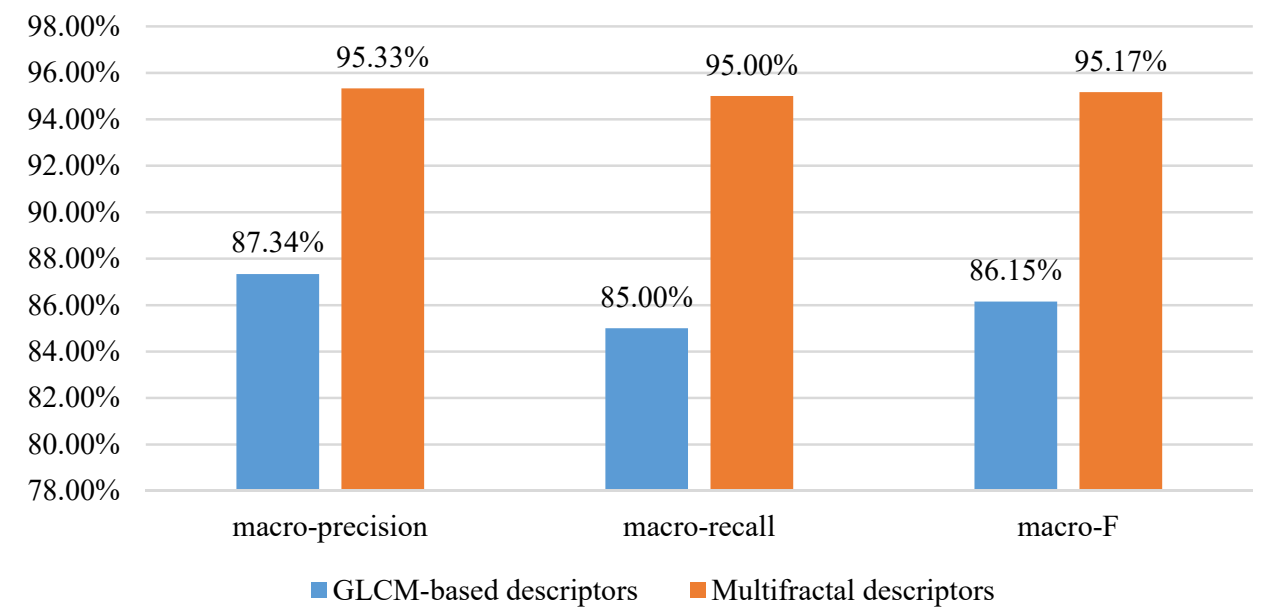

Figure 12. Average performance evaluation of different texture descriptors. 
Table 4. Performance of inertinite macerals' classification with multifractal descriptors.

\begin{tabular}{ccccccccc}
\hline & Pyrofusinite & Oxyfusinite & Semifusinite & Secretinite & Funginite & Macrinite & Inertodetrinite & Micinite \\
\hline precision & 1.0000 & 0.8696 & 1.0000 & 0.9048 & 0.9000 & 1.0000 & 0.9524 & 1.0000 \\
recall & 0.8500 & 1.0000 & 0.9500 & 0.9500 & 0.9000 & 1.0000 & 1.0000 & 0.9500 \\
F-measure & 0.9189 & 0.9302 & 0.9744 & 0.9268 & 0.9000 & 1.0000 & 0.9756 & 0.9744 \\
\hline
\end{tabular}

Table 5. Performance of inertinite macerals' classification with GLCM-based descriptors.

\begin{tabular}{ccccccccc}
\hline & Pyrofusinite & Oxyfusinite & Semifusinite & Secretinite & Funginite & Macrinite & Inertodetrinite & Micinite \\
\hline precision & 0.8182 & 0.9756 & 0.9231 & 0.7368 & 0.7083 & 0.9756 & 0.6667 & 0.9744 \\
recall & 0.9000 & 1.0000 & 0.9000 & 0.7000 & 0.8500 & 1.0000 & 0.5000 & 0.9500 \\
F-measure & 0.8182 & 0.9756 & 0.9231 & 0.7368 & 0.7083 & 0.9756 & 0.6667 & 0.9744 \\
\hline
\end{tabular}

\section{Conclusions}

Considering the fact that the petrological properties of coal are complex and widely distributed, in this paper, the microscopic images with heterogeneous natural have been analyzed by the MF-DFA method. We verified the multifractal properties of the microscopic image by the function of $\tau(q)$ and $h(q)$. In addition, with the multifractal spectrum, we have proposed three important texture descriptors for characterizing image information, such as $\alpha_{\min }, \alpha_{\max }$, and $f_{\max }$. It is well known that the texture descriptor of an image should be robust and immune to image quality; thus, the stability experiments have been implemented and the results have verified the anti-noise ability and anti-blur capability of the multifractal descriptors.

A classification model with RBF-SVM classifier has been built to distinguish the 160 microscopic images of inertinite macerals in coal. Our multifractal descriptors have represented the most appealing results in terms of performance metrics of precision, recall, and F-measure, providing excellent performance compared with GLCM-based texture descriptors. The successful implementation of our proposed method in the identification of inertinite materials can assist petrologists to make correct decisions and reduce the influences of subjective factors in practical scenarios, which is particularly beneficial to geologists with less experience. In view of the fact that there are some similarities of structural complicacy and non-linear multi-classification, we will investigate the classification of other maceral groups with a reference to our proposed method in the future. Simultaneously, in order to be more suitable for industrial applications, we will also develop a cross platform software for maceral image recognition and classification in the future work.

Author Contributions: Conceptualization, P.W. and M.L.; formal analysis, M.L.; investigation, M.L and S.C.; resources, D.Z.; data curation, D.Z.; writing—original draft preparation, M.L.; writing—review and editing, P.W.; supervision, S.C.; project administration, P.W.; funding acquisition, P.W.

Funding: This research was funded by the National Natural Science Foundation of China (number 51574004); Natural Science Foundation of the Higher Education Institutions of Anhui Province, China (number KJ2019A0085); Academic Foundation for Top Talents of the Higher Education Institutions of Anhui Province, China (number 2016041).

Conflicts of Interest: The authors declare no conflict of interest.

\section{References}

1. Flores, B.D.; Borrego, A.G.; Diez, M.A.; da Silva, G.L.; Zymla, V.; Vilela, A.C.; Osório, E. How coke optical texture became a relevant tool for understanding coal blending and coke quality. Fuel Process. Technol. 2017, 164, 13-23. [CrossRef]

2. Piechaczek, M.; Mianowski, A.; Sobolewski, A. Reprint of "The original concept of description of the coke optical texture". Int. J. Coal Geol. 2015, 139, 184-190. [CrossRef]

3. Chalmers, G.R.; Bustin, R.M. A multidisciplinary approach in determining the maceral (kerogen type) and mineralogical composition of Upper Cretaceous Eagle Ford Formation: Impact on pore development and pore size distribution. Int. J. Coal Geol. 2017, 171, 93-110. [CrossRef] 
4. Yang, J.; Stansberry, P.G.; Zondlo, J.W.; Stiller, A.H. Characteristics and carbonization behaviors of coal extracts. Fuel Process. Technol. 2002, 79, 207-215. [CrossRef]

5. Wang, H.; Lei, M.; Chen, Y.; Li, M.; Zou, L. Intelligent identification of maceral components of coal based on image segmentation and classification. Appl. Sci. 2019, 9, 3245. [CrossRef]

6. Mlynarczuk, M.; Skiba, M. The application of artificial intelligence for the identification of the maceral groups and mineral components of coal. Comput. Geosci. 2017, 103, 133-141. [CrossRef]

7. D'Angelo, G.; Palmieri, F.; Rampone, S. Detecting unfair recommendations in trust-based pervasive environments. Inf. Sci. 2019, 486, 31-51. [CrossRef]

8. Lester, E.; Watts, D.; Cloke, M. A novel automated image analysis method for maceral analysis. Fuel 2002, 81, 2209-2217. [CrossRef]

9. Ruan, X.D.; ZHAO, W.F. Recognizing of overlapped coal particles in microscope images. J. China Coal Soc. 2005, 30, 769-771.

10. Wang, P.Z.; Ding, H.T.; Liu, C.L. Coal microscope image contour extraction algorithm based on structuring elements. J. China Coal Soc. 2014, 39, 285-288. [CrossRef]

11. Wang, S.; Zhu, X.K.; Lyu, Q. Coal rock macerals recognition based on RILBP-GLCM algorithm. J. China Coal Soc. 2017, 36, 142-144.

12. Wang, P.Z.; Yin, Z.H.; Wang, G. A classification method of vitrinite for coal macerals based on the PCA and RBF-SVM. J. China Coal Soc. 2017, 42, 977-984.

13. Wang, P.Z.; Reng, J.; Du, C.L. Classification of macerals in exinite of coal based on Tamura features. J. Anhui Univ. Technol. (Nat. Sci. Ed.) 2018, 35, 131-136.

14. Wang, P.Z.; Liu, J.M.; Wang, W.Y. Classification of macerals in exinite of coal based on contourlet transform. J. China Coal Soc. 2018, 43, 641-645.

15. Wang, P.Z.; Wang, H.; Liu, M. A PCA-SLPP dimensionality reduction method based on manifold learning. J. Anhui Univ. Technol. (Nat. Sci. Ed.) 2018, 35, 352-359.

16. Mandelbrot, B. How long is the coast of Britain? Statistical self-similarity and fractional dimension. Science 1967, 156, 636-638. [CrossRef] [PubMed]

17. Mahamud, M.; Óscar, L.; Pis, J.J.; Pajares, J.A. Textural characterization of coals using fractal analysis. Fuel Process. Technol. 2003, 86, 135-149. [CrossRef]

18. Nie, B.; Liu, X.; Yang, L.; Meng, J.; Li, X. Pore structure characterization of different rank coals using gas adsorption and scanning electron microscopy. Fuel 2015, 158, 908-917. [CrossRef]

19. Liu, X.F.; Nie, B.S. Fractal characteristics of coal samples utilizing image analysis and gas adsorption. Fuel 2016, 182, 314-322. [CrossRef]

20. Li, Y.Y.; Zhang, S.C.; Xin, Z. Classification and fractal characteristics of coal rock fragments under uniaxial cyclic loading conditions. Arabian J. Geosci. 2018, 11, 201. [CrossRef]

21. Pandey, R.; Harpalani, S. An imaging and fractal approach towards understanding reservoir scale changes in coal due to bioconversion. Fuel 2018, 230, 282-297. [CrossRef]

22. Kantelhardt, J.W. Multifractal detrended fluctuation analysis of nonstationary time series. Phys. A 2002, 316, 87-114. [CrossRef]

23. Zhao, H.; He, S. Analysis of speech signals' characteristics based on MF-DFA with moving overlapping windows. Phys. A 2016, 442, 343-349. [CrossRef]

24. Rizvi, S.A.R.; Dewandaru, G.; Bacha, O.I.; Masih, M. An analysis of stock market efficiency: Developed vs Islamic stock markets using MF-DFA. Phys. A 2014, 407, 86-99. [CrossRef]

25. Mensi, W.; Tiwari, A.K.; Yoon, S.M. Global financial crisis and weak-form efficiency of Islamic sectoral stock markets: An MF-DFA analysis. Phys. A 2017, 471, 135-146. [CrossRef]

26. Mukhopadhyay, S.; Mandal, S.; Das, N.K.; Dey, S.; Mitra, A.; Ghosh, N.; Panigrahi, P.K. Diagnosing heterogeneous dynamics for CT scan images of human brain in wavelet and MFDFA domain. In Advances in Optical Science and Engineering; Springer: Berlin, Germany, 2015; pp. 335-340.

27. Sỳkorová, I.; Pickel, W.; Christanis, K.; Wolf, M.; Taylor, G.; Flores, D. Classification of huminite-ICCP System 1994. Int. J. Coal Geol. 2005, 62, 85-106. [CrossRef]

28. Scott, A.C.; Glasspool, I.J. Observations and experiments on the origin and formation of inertinite group macerals. Int. J. Coal Geol. 2007, 70, 53-66. [CrossRef]

29. Gu, G.F.; Zhou, W.X. Detrended fluctuation analysis for fractals and multifractals in higher dimensions. Phys. Rev. E Stat. Nonlinear Soft Matter Phys. 2006, 74, 061104. [CrossRef] 
30. Meakin, P. Fractals, Scaling and Growth Far from Equilibrium; Cambridge University Press: London, UK, 1998; Volume 5 .

31. Chen, Y.X.; Wang, J.Z. A region-based fuzzy feature matching approach to content-based image retrieval. IEEE Trans. Pattern Anal. Mach. Intell. 2002, 24, 1252-1267. [CrossRef]

32. Zhu, X.; Li, N.; Pan, Y. Optimization Performance Comparison of Three Different Group Intelligence Algorithms on a SVM for Hyperspectral Imagery Classification. Remote Sens. 2019, 11, 734. [CrossRef]

33. Wang, T.Y.; Chiang, H.M. One-against-one fuzzy support vector machine classifier: An approach to text categorization. Expert Syst. Appl. 2009, 36, 10030-10034. [CrossRef]

34. Kang, S.; Cho, S.; Kang, P. Constructing a multi-class classifier using one-against-one approach with different binary classifiers. Neurocomputing 2015, 149, 677-682. [CrossRef]

(C) 2019 by the authors. Licensee MDPI, Basel, Switzerland. This article is an open access article distributed under the terms and conditions of the Creative Commons Attribution (CC BY) license (http:// creativecommons.org/licenses/by/4.0/). 